\title{
Endometrial stromal tumor with sex-cord-like elements
}

\author{
Katarzyna Wojda, Piotr Sieroszewski
}

Department of Fetal Medicine and Gynecology First Chair of Gynecology and Obstetrics, Medical University of Lodz, Poland

A 42-year-old woman was admitted to the Tertiary Obstetrics and Gynecology Care Center due to the suspicion of a uterine cyst. The patient was nulligravida, never miscarried and reported regular but profuse menstruations.

The transvaginal ultrasound showed the anteflexion of uterus sized $94 \times 77 \times 70 \mathrm{~mm}$, with a hypoechogenic clearly limited cyst in the size of $54 \times 64 \times 64 \mathrm{~mm}$ with thin septa inside, modeling the uterine cavity, nevertheless, having no contact with the cavity as shown in the ultrasound (Fig. 1, 2). Inside the lump, no pathological vascularization was found in Color and Power Doppler. The endometrial thickness was $17 \mathrm{~mm}$ (Fig. 3). The ovaries seemed to be normal in the ultrasound scan.

CA 125 concentration was normal, inflammatory markers and $\beta$-hCG were negative.

The patient was qualified for a diagnostic hysteroscopy in order to determine the topography of the lesion and to exclude its location inside the uterine cavity. The hysteroscopy found that the uterine cavity was normal. The patient was qualified for transverse Pfannenstiel approach and enucleation of the lesion from the uterus with intraoperative pathological examination.

The lesion was excised undamaged. Intraoperative pathological examination indicated suspicious lesion, advising final immunohistological verification. A tumor of the uterus was diagnosed, described as cell-rich, solid, consisting of oval rather monomorphic cells without major features of nuclear atypia. The tumor had well-defined borders separating it from the muscle, with no signs of necrosis, with rather numerous cellular divisions. The immunohistological study determined the co-expression of broad spectrum of cytokeratin and actin, with lack of expression inhibin, Melan-A and $\mathrm{H}$-caldesmon. The morphological image, co-expression of cytokeratin and actin resembled the UTROSCT (uterine tumor resembling ovarian sex-cord tumors), however, with lacking expression of inhibin and Melan-A, as well as increased mitotic index, the image pointed more towards ESTSCLE (endometrial stromal tumor with sex-cord like elements).

The patient was again admitted to the Department and, after oncological consultation she was qualified for radical hysterectomy and possible further treatment. The pathological study did not reveal any anomalies, the uterus did not show any features of neoplastic growth. Currently, after three years from the radical hysterectomy patient is in good condition having no pathological symptoms.

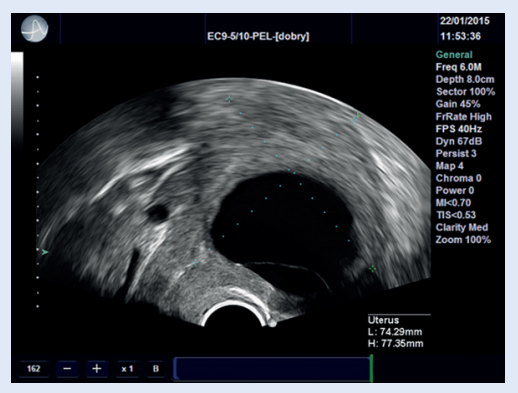

Figure 1. Median sagittal section of the uterus

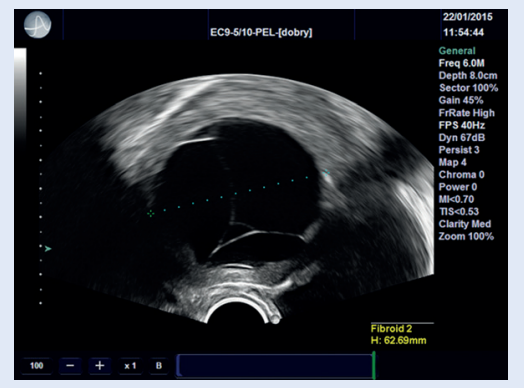

Figure 2. The diameter of the cystic structure of the uterus.

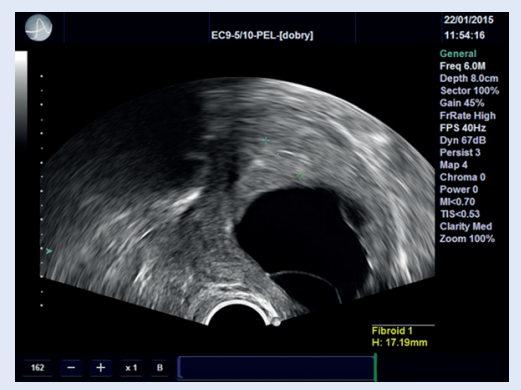

Figure 3. Endometrium thickness 